\title{
Dynamic surface behaviour of VPO catalysts under reactive and non- reactive gas compositions: an in-situ XAS study
}

\author{
M. Hävecker*, A. Knop-Gericke, H. Bluhm, E. Kleimenov, R.W. Mayer ${ }^{+}$, M. Fait ${ }^{\#}$, R. Schlögl,
}

Department of Inorganic Chemistry, Fritz-Haber-Institute of the MPG, Faradayweg 4-6, 14195 Berlin, Germany

${ }^{+}$Degussa, Project House Catalysis, Industriepark Höchst, D-65926 Frankfurt / M, Germany

\# Institut für Angewandte Chemie Berlin-Adlershof e.V., Richard-Willstätter-Strasse 12, D-12489 Berlin, Germany

* Corresponding author: e-mail mh@,fhi-berlin.mpg.de, tel.: +49 308413 4422, fax: +49 3084134677

accepted 17 April 2004

\begin{abstract}
The surface of an activated vanadium phosphorus oxide (VPO) catalyst was investigated by means of in situ X-ray absorption spectroscopy in the total electron yield mode. We observed significant changes of the $\mathrm{V} \mathrm{L}_{3}$-near edge X-ray absorption fine structure (NEXAFS) when the material was transferred from room temperature to working conditions at $400^{\circ} \mathrm{C}$ in the reaction atmosphere. We studied the same VPO material under different gas compositions comprising the reaction mixture of $n$-butane and oxygen, pure oxygen and vacuum to elucidate the influence of the gas-surface interaction and the effect of the temperature. The results of this extensive study indicate a dynamic response of the catalyst surface to the applied conditions.
\end{abstract}

Keywords: vanadium phosphorus oxide (VPO); X-ray absorption spectroscopy (XAS); vanadium L3-edge; n-butane oxidation; electronic structure

\section{Introduction}

Bulk vanadium phosphorus oxide (VPO) catalysts are industrially used for the production of maleic anhydride (MA) from $n$-butane $[1,2]$. The VPO system has been intensively studied for the last three decades, but still insufficient information is available about the exact nature of the catalytic active site [3-7]. Especially, there is a lack of surface sensitive characterisation of the working catalyst, which is essential for the understanding of the catalytic operation of a non-supported active phase. It is clear that surface chemistry and the bulk properties of the catalyst change during the activation [8-11]. The composition of the VPO surface is complex since crystalline phases other than the main vanadyl pyrophosphate are often observed and also disordered phases are known to exist [12-14].

Recently, we reported on the state of the electronic surface structure of a VPO catalyst prepared in aqueous solution under $n$-butane oxidation conditions [15]. We used in situ X-ray absorption spectroscopy (XAS) in the soft en- ergy range ( $\mathrm{h} v \leq 1000 \mathrm{eV}$ ) for spectroscopic characterisation. XAS in the soft energy range is useful in the context of the present problem because it is surface sensitive when operated in the electron yield mode and can be applied in situ. The $\mathrm{V} \mathrm{L}_{3}$-near edge X-ray absorption fine structure (NEXAFS) is very sensitive to the local electronic structure at the $\mathrm{V}$ site that is supposed to be related to the catalytic activity of the material. In this paper we extend our earlier work and present a study of VPO catalysts prepared by the alcoholic route. Thereby, the significance of our findings for the VPO system in general will be stressed. We investigate in more detail the response of one catalyst on varying gas atmospheres (oxidising / reducing character) to elucidate the changes of the electronic surface structure under these different conditions. The influence of the X-ray beam on the sample surface will be discussed, too. Hence, in this paper we present an extensive study of the VPO surface under various conditions. 


\section{Experimental}

\subsection{Catalyst preparation and testing}

Vanadium phosphate catalyst precursors were prepared by reduction of $\mathrm{V}_{2} \mathrm{O}_{5}$ (Merck) with isobutyl alcohol in a phosphoric/phosphorous acid medium (85\% Aldrich). The precursors were pelleted ( $9 \mathrm{MPa}, 0.5 \mathrm{~min})$, crushed, and the sieve fraction 1.25 to $2.5 \mathrm{~mm}$ was transformed to the fresh catalyst in a tubular reactor $\left(11 \mathrm{~mm}\right.$ i.d.) at $400{ }^{\circ} \mathrm{C}$ in $1 \mathrm{vol} \%$ $\mathrm{O}_{2}$ in $\mathrm{N}_{2}$. The fresh catalyst was conditioned in the same reactor by heating the material in a mixture of air and $n$ butane. The gases were fed to the reactor via calibrated mass flow controllers to give a feedstock composition of varying $n$-butane concentrations in air $\left(0.5 \%-1.5 \%\right.$, GSHV: $\left.600 \mathrm{~h}^{-1}\right)$ at $400{ }^{\circ} \mathrm{C}$. The products were then fed via a heated capillary to an on-line gas chromatograph for product analysis. A thermocouple was located in the centre of the catalyst bed and temperature control was better than $\pm 1^{\circ} \mathrm{C}$. Carbon mass balances of $\geq 97 \%$ were typically observed. After $200 \mathrm{~h}$ of activation a steady selectivity of $64 \%$ to MA at a $n$-butane conversion of $80 \%$ was obtained.

\subsection{Characterisation}

After testing the catalyst in the tubular reactor the catalyst powder $(50 \mathrm{mg})$ was pressed into a self supporting flat pellet (diameter $13 \mathrm{~mm}$ ) and mounted on a stainless steel sample holder for further characterisation by in situ X-ray absorption spectroscopy. The XAS experiments were performed using a reactor cell especially designed for in situ soft XAS investigations. Details about the set-up and the data processing can be found in the literature [16-18]. For in situ experiments a continuous flow of 1.5 vol $\% n$-butane in helium balance and oxygen was dosed via two calibrated mass flow controllers at a total mass flow of $35 \mathrm{sccm}$. The partial pressures of the $n$-butane / helium mixture and the oxygen were 1.6 mbar and 0.4 mbar, respectively, resulting in a total pressure in the reactor of 2 mbar during the XAS measurements. Furthermore, experiments were carried out under pure oxygen ( $\mathrm{p}=0.4$ mbar) and under vacuum conditions ( $\mathrm{p}=1 \cdot 10^{-7} \mathrm{mbar}$ ) as well. The sample was heated up to $400^{\circ} \mathrm{C}$ by a resistive heater. The sample temperature was held at $400{ }^{\circ} \mathrm{C}$ for typically $80 \mathrm{~min}$ (in the case of pure oxygen $180 \mathrm{~min}$ ) and then decreased to room temperature.

Reference materials $\mathrm{VOPO}_{4} * 2 \mathrm{H}_{2} \mathrm{O}, \beta-\mathrm{VOPO}_{4}$ and $\mathrm{V}_{2} \mathrm{O}_{5}$ were characterized by XAS, too. The powders were fixed on the sample holder by double adhesive conducting tape. The phase composition of these materials was verified by XRD prior to the XAS studies.

Experiments were carried out at the undulator beamline U49-2 / PGM1 at the third generation synchrotron radiation facility BESSY in Berlin [19, 20]. The data were collected in situ in the total electron yield mode (TEY) under gas flow conditions. The photon energy of the NEXAFS spectra was calibrated by the $\pi^{*}$-resonance of molecular oxygen at $530.8 \mathrm{eV}[21]$. The resolving power $\mathrm{E} / \Delta \mathrm{E}$ was about 5000 .

\section{Results and discussions}

In Fig. 1 the $\mathrm{V} \mathrm{L}_{3}$-edge spectra (corresponding to $\mathrm{V} 2 \mathrm{p}_{3 / 2} \rightarrow \mathrm{V} 3 \mathrm{~d}$ electronic transitions) taken in a $n-\mathrm{C}_{4} \mathrm{H}_{10} / \mathrm{O}_{2}$ mixture (upper spectrum), under vacuum (middle spectrum) and in pure $\mathrm{O}_{2}$ (lower spectrum) are shown for $400{ }^{\circ} \mathrm{C}$ (left panel) and after the heating procedure at $25^{\circ} \mathrm{C}$ (right panel).
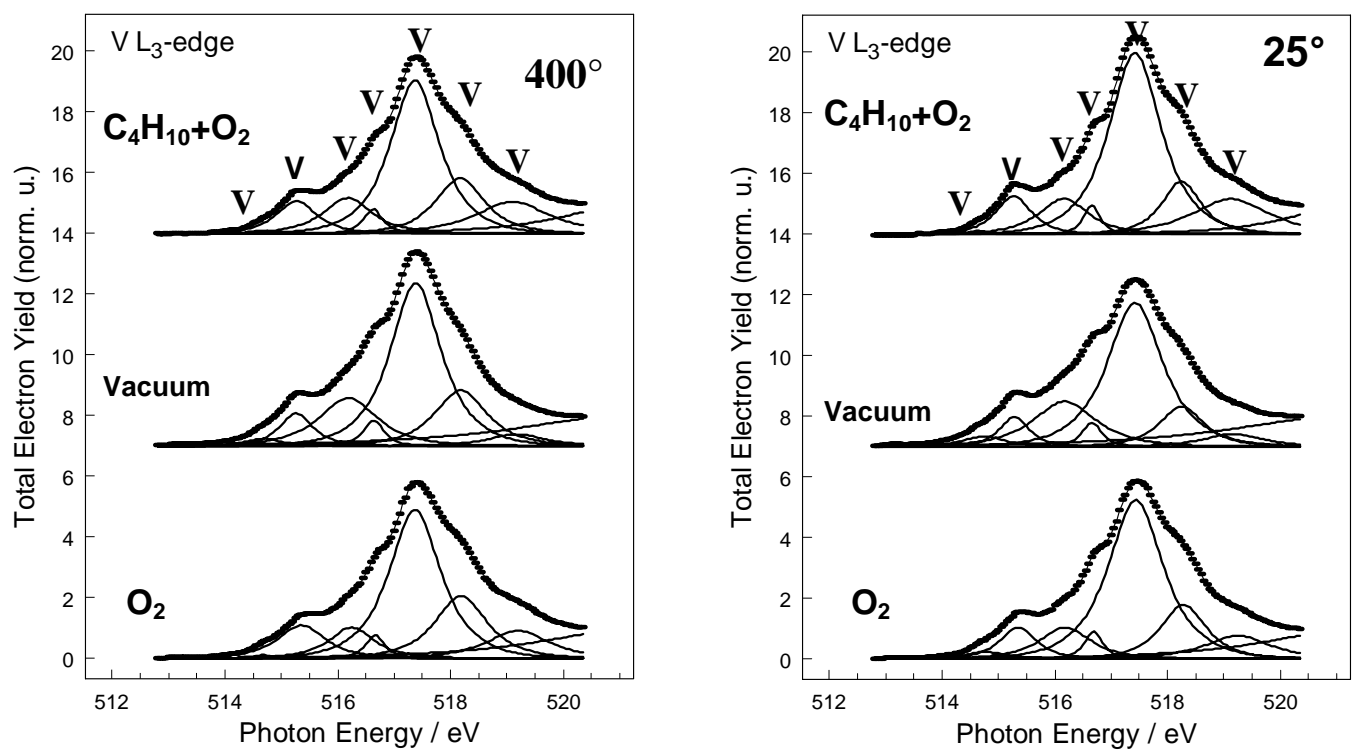

Figure 1: In situ $\mathrm{V} \mathrm{L}_{3}$-NEXAFS of VPO at $400{ }^{\circ} \mathrm{C}$ (left panel) and after heating at $25^{\circ} \mathrm{C}$ (right panel) in $\mathrm{C}_{4} \mathrm{H}_{10}+\mathrm{O}_{2}$, vacuum, and $\mathrm{O}_{2}$. The spectra have been background subtracted and normalised to the same edge height. 
Vanadium $\mathrm{L}_{3}$-edge spectra were normalised to the same absorption edge height at $522 \mathrm{eV}$ after subtraction of a constant background and analysed by a least squares fit, using Gauss-Lorentz profiles considering experimental and intrinsic broadening. Seven fit profiles are necessary to achieve an adequate simulation of the NEXAFS. These are also shown in Fig. 1 and labelled as V1-V7. The spectral weights of the peaks V1-V7 have been determined for several spectra under the same conditions and averaged. These values were used as primary experimental data.

In order to judge the accuracy of the measurements and the significance of subtle changes of the NEXAFS we evaluated the error of our data analysis. The fit is robust against variations of the initial fit parameters as long as the number of fit profiles was kept constant. The minimum number of fit profiles to get a satisfying, unique fit was used. The signal to noise ratio of the experimental data is very high. The data show no visible noise. The background is constant in the energy range of the $\mathrm{V} \mathrm{L}_{3}$-edge. A variation of the background was only observed during heating ramps. Therefore, only data recorded at thermal equilibrium were considered for further data analysis to exclude this source of systematic error. Several measurements were carried out under the same experimental conditions, analysed, and the resulting resonance intensities have been averaged. The variation of the single data has been used for the estimation of the statistical error of the mean. The $90 \%$ double-sided confidence interval has been determined according to $\bar{x} \pm t_{0.9, f} \cdot \frac{\sigma}{\sqrt{n}}$, where $\bar{x}$ is the mean, $\sigma$ the standard deviation, $\mathrm{n}$ the number of measurements and $t_{0.9, f}$ the tabulated Student $\mathrm{t}$-factor for a $90 \%$ confidence interval and $\mathrm{f}$ degrees of freedom (i.e. $\mathrm{n}-1$ in this case). We used Student's t-distribution rather than the normal distribution because this should give a more justified (i.e. unbiased) value for the error for a small number of data points $(n<30)$. An error bar indicates the confidence interval in the figures. The error of the single average values is different due to different standard deviations and number of the single measurements. In general, it can be concluded that the measurements and the data processing have a high accuracy. Thus, detailed information can be gained from the experimental data.

\section{1. n-Butane oxidation conditions}

The analysis of the contribution of different features in the $\mathrm{V} \mathrm{L}_{3}$-NEXAFS to the total intensity revealed significant changes when the catalyst was heated from room temperature to the reaction temperature of $400{ }^{\circ} \mathrm{C}$ in a mixture of $n$-butane and oxygen. We found a decrease of the relative spectral intensity of resonance V5 (89\% and $86 \%$, respectively, of the initial value) and a strong increase of the spectral weight of resonance V6 (148\% and 159\%, respectively) when the temperature was increased to $400{ }^{\circ} \mathrm{C}$ (Fig. 2A, B). These changes in the electronic surface struc

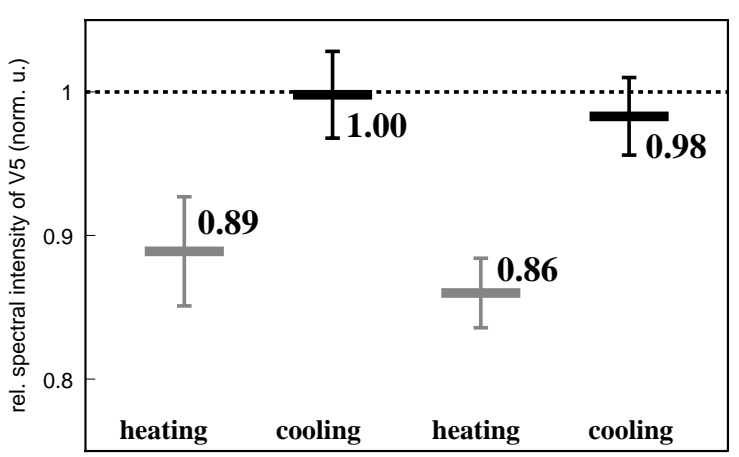

conditions

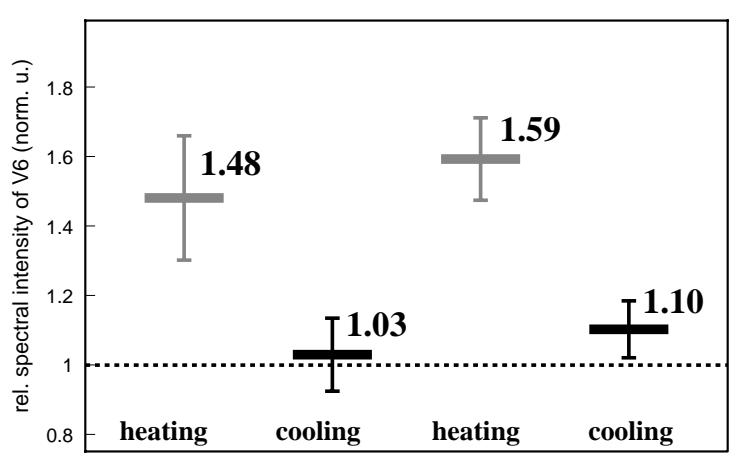

conditions

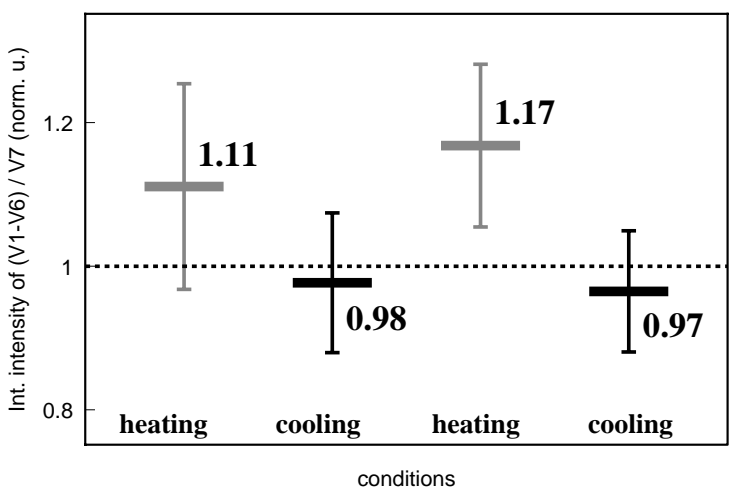

Figure 2: Normalised averaged value of the integrated intensity of V5 (A), V6 (B) and the ratio of the integrated intensities of the sum V1-V6 to V7 (C). The catalyst was heated to $400{ }^{\circ} \mathrm{C}$ (heating) and cooled down to room temperature (cooling) in a mixture of $n$-butane and oxygen. The initial intensity of the resonances prior to the first heating procedure was taken as 1 (dashed line). The error bar indicates the $90 \%$ confidence interval of the data point.

ture were almost completely reversible when the primary conditions were re-established. The photon energy position of resonances V5 and V6 shifted to slightly lower values at the same time. This energy position change was reversible, too. This modification of the electronic structure at the vanadium site of this sample derived by the alcoholic preparation route is completely consistent with our previous observations for VPO catalysts prepared by the aqueous route [15]. 
These findings clearly indicate that the surface electronic structure undergoes significant rearrangements when going from room temperature to working conditions at 400 ${ }^{\circ} \mathrm{C}$ in the reaction atmosphere of $n$-butane and oxygen.

\subsection{Irradiation stability}

One reason for the observed surface modification could be damage by the incident photon beam. VPO catalysts are known for their sensitivity to irradiation by high energetic electrons used in TEM. Usually, both the surface morphology of this material and the electronic structure is
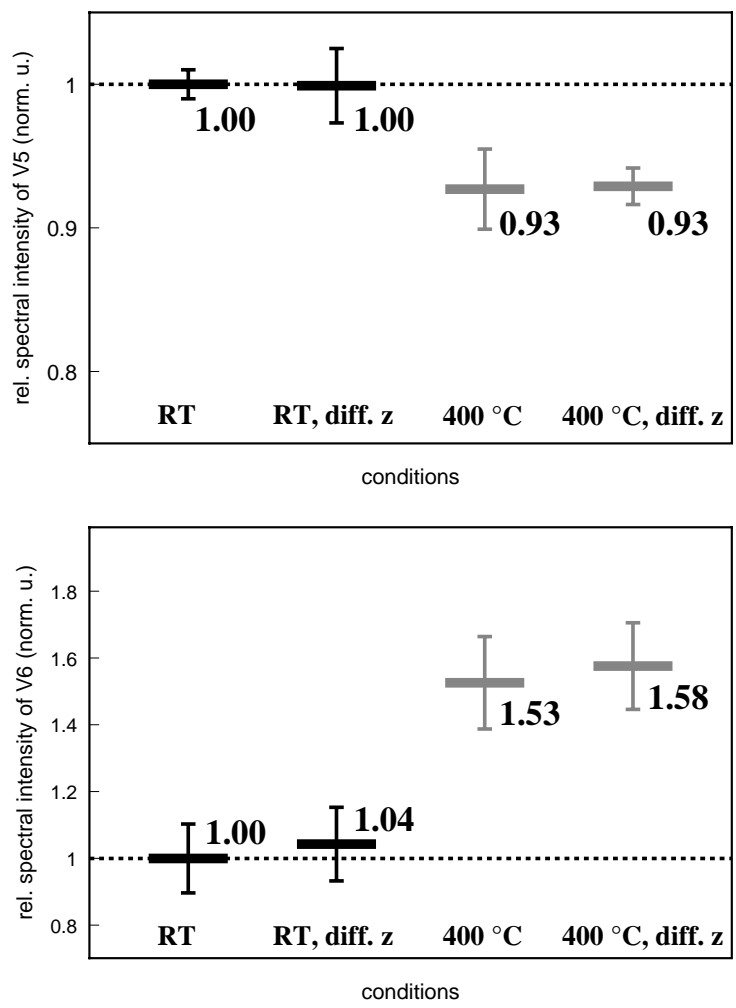

Figure 3:Normalised averaged value of the integrated intensity of V5 (A) and V6 (B). The resonance intensity was determined at room temperature and $400{ }^{\circ} \mathrm{C}$ for 2 different lateral positions on the surface (different $\mathrm{z}$ ). The initial intensity of the resonances prior to the heating procedure was taken as 1 (dashed line). The error bar indicates the $90 \%$ confidence interval of the data point.

modified when it is studied by HRTEM. The interaction of soft X-rays with the material is much weaker. Nevertheless, with the advent of high-brilliance synchrotron radiation sources like BESSY II with their high brilliant X-ray beams $\left(10^{18}\right.$ photons $\left./\left(\mathrm{s} \mathrm{mm}^{2} \mathrm{mrad}^{2}\right)\right)$ a destruction of sample surfaces is sometimes observed. We made specific experimental arrangements to take care of this source of surface modification that would not be related to the interaction with the gaseous atmosphere. We aligned the sample position out of the focus of the beamline. This caused an enlargement of the spot size from ca. $70 \mu \mathrm{m}$ to roughly 2 $\mathrm{mm}$ in the horizontal direction and therefore an approximately 30 times reduced photon density because of the divergence of the X-ray beam. It can be concluded that the $\mathrm{X}$-ray beam in our in situ experiments did not alter the electronic surface structure of the catalyst for mainly two reasons. Firstly, we proved that the changes we observed under reaction conditions are reversible. Secondly, we measured different lateral positions on the surface in vacuum. The sample was moved vertically, perpendicular to the X-ray beam after one part of the surface had been irradiated for a prolonged time (i.e. $90 \mathrm{~min}$ ). It could easily be ensured that the X-ray beam hit a different area of the sample because the size of the spot was only a few hundred micrometers in this direction. The result of this experiment is shown in Fig. 3. The same relative intensity of resonance V5 and V6 was found at different positions (i.e. a spot with prolonged irradiation and a fresh spot) on the catalyst surface. This proves that changes of the NEXAFS under reaction conditions did not occur due to beam damage in the time scale of the experiment. It took about $8 \mathrm{~min}$ to record a high resolution NEXAFS spectrum. Furthermore, it shows that the sample surface was homogeneous on the spatial resolution scale of our experiment. The mean of the two measurements at the same temperature for different sample positions remained very well inside the statistically estimated $90 \%$ confidence interval. This is an indication that the real error might be overestimated.

\subsection{Influence of the gas phase and the tem- perature}

From the previous it becomes clear that beam damage did not cause the surface modifications described in section 3.1. In the following the influence of the temperature and of the gas phase composition on the surface will be discussed. In general, the NEXAFS depends on the temperature of the material. This is due to the influence of lattice thermal expansion and atomic vibrational motion on the NEXAFS. A broadening of the resonances and therefore a "smearing out" of the fine structure was observed. The relative position of certain resonances was altered for different temperatures in the case of $\mathrm{KCl}[22,23]$. However, these modifications caused solely by temperature changes are very minor.

In order to study the influence of the gas phase composition and the temperature on the VPO surface, we exposed the same catalyst material to a mixture of $n$-butane and oxygen (plus helium as balance), as well as to pure oxygen and to vacuum conditions. At each condition we cycled the temperature from room temperature to $400{ }^{\circ} \mathrm{C}$ and back to $25^{\circ} \mathrm{C}$. The $\mathrm{V} \mathrm{L}_{3}$-NEXAFS was analysed in the same way as described before. The results are summarised in Fig. 4.

A decrease of the relative intensity of $\mathrm{V} 5$ at the $\mathrm{V} \mathrm{L}_{3}$ edge upon heating to $400{ }^{\circ} \mathrm{C}$ (light bar) could be observed under all conditions (Fig. 4A). But while this intensity change is small under vacuum conditions $(93 \%$ of the ini- 
tial value), the intensity decrease was significantly more pronounced when the catalyst was heated in the $n$-butane reaction mixture or in pure oxygen $(86 \%$ of the initial value in both cases). Furthermore, when the catalyst was cooled back to room temperature (dark bar), the intensity
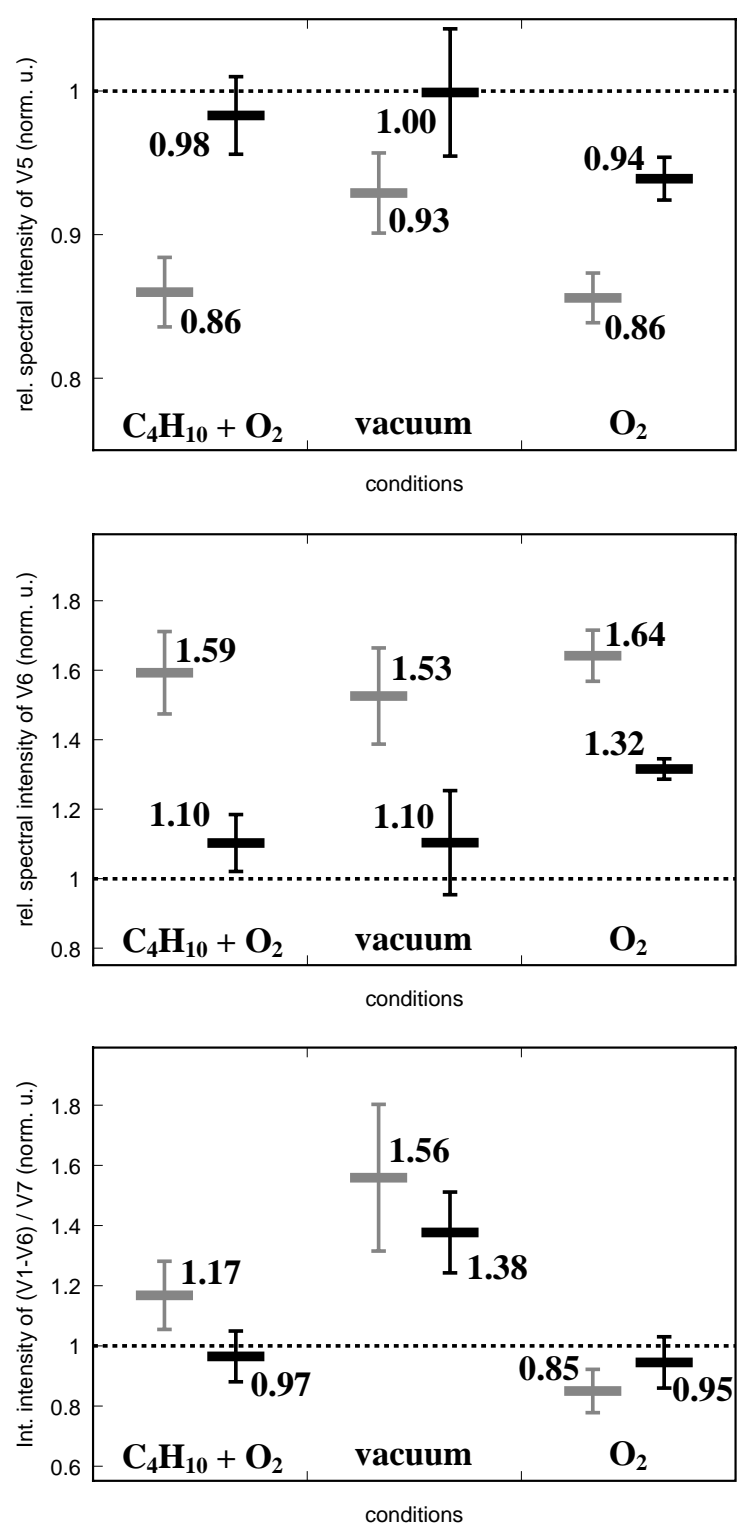

Figure 4: Normalised averaged value of the integrated intensity of V5 (A), V6 (B) and the ratio of the integrated intensities of the sum V1-V6 to V7 (C). The catalyst was heated to $400{ }^{\circ} \mathrm{C}$ (light bar) and cooled down to room temperature (dark bar) under different conditions: $\mathrm{C}_{4} \mathrm{H}_{10}+\mathrm{O}_{2}$, vacuum, and $\mathrm{O}_{2}$. The initial intensity of the resonances prior to the heating procedure was taken as 1 (dashed line). The error bar indicates the $90 \%$ confidence interval of the data point.

change observed under $n$-butane / oxygen mixture was reversible (98\% of the initial value) while a slightly smaller value (94\%) was found for pure oxygen. This result clearly indicates the influence of the gas phase on the intensity changes of resonance V5.
A strong increase of the spectral weight of V6 was observed under reaction conditions (Fig. 2B). The V6 intensity increased when the material was heated in pure oxygen and in vacuum, too. These data can be found in Fig. 4B. The V6 intensity mean suggests a most pronounced increase (164\% of the initial value) when the catalyst was heated in pure oxygen, although the determined intensities overlap under all tested conditions taking the $90 \%$ confidence interval into account. Adsorbed reactants and intermediates on the surface influence the intensity of V6. This was concluded in [15] from the coincidence of desorption traces of maleic anhydride, dihydrofuran, furan and butene with the resonance intensity variation. The most pronounced intensity change is expected in an oxygen atmosphere because gas phase oxygen could be used for the oxidation of the adsorbates that subsequently desorb from the surface.

A substantial difference in the response on the heating conditions was observed when the catalyst was cooled down again. While the intensity change of V6 was almost completely reversible under vacuum conditions and in the $n$-butane reaction mixture, the relative intensity of V6 was still increased to $132 \%$ of its initial value in pure oxygen. The reversibility of the V6 intensity change when the catalyst is cooled in vacuum was rather unexpected. The V6 intensity should be preserved under vacuum, if the changes where solely caused by adsorbed molecules. Therefore it can be concluded that there are also other factors influencing the spectral weight of this resonance. The intensity variations in the case of pure oxygen treatment will be discussed later.

Additionally, we estimated the spectral weight that can be assigned to $t_{2 \mathrm{~g}}$-like unoccupied states relative to the weight of the $e_{\mathrm{g}}$-like unoccupied states to get a more profound understanding of the character of the vanadiumoxygen bond. The denotation $\mathrm{t}_{2 \mathrm{~g}}$ and $\mathrm{e}_{\mathrm{g}}$ for electron orbitals is used frequently, although it is strictly valid only in $\mathrm{O}_{h}$ symmetry and not for the case of the highly distorted $\mathrm{VO}_{6}$ octahedron [24]. In $\mathrm{O}_{\mathrm{h}}$ symmetry the $\mathrm{t}_{2 \mathrm{~g}}$ states are the irreducible representation of the bonds with $\pi$-character and the $e_{g}$ states are the irreducible representation of the bonds with $\sigma$-character. The unique identification of states with $\mathrm{t}_{2 \mathrm{~g}}$ and $\mathrm{e}_{\mathrm{g}}$ character is not possible without a detailed calculation of the density of states. Unfortunately, this is not available for VPO at the moment. The theoretical description of the $\mathrm{V} \mathrm{L}_{3}$-edge electronic structure of VPO is hindered by the complex geometric structure of the material and by the strong electron correlation caused by the localised electron system. We chose a semi-empirical approach to get this valuable information about the V-O bond character. We showed in a previous work that the NEXAFS of $\mathrm{V}_{2} \mathrm{O}_{5}$ and VPO exhibit certain similarities [25]. This is probably due to their resembling local geometric structure. Vanadium atoms are centred in highly distorted octahedra in both compounds. Density functional theory calculations of the electronic structure of $\mathrm{V}_{2} \mathrm{O}_{5}$ showed that the $\mathrm{t}_{2 \mathrm{~g}}$ and $e_{g}$ states are clearly separated at the vanadium site in this kind of material, with the $e_{g}$ states following the $t_{2 g}$ states 
on the high photon energy side [26]. It is not well defined at which photon energy the states with mainly $\mathrm{e}_{\mathrm{g}}$ character start to dominate the NEXAFS. Following the reasoning in [25] based on an empirical bond length / resonance position relationship we assumed that all intensity up to resonance V6 is dominated by $t_{2 g}$ character. This seems to be justified because V7 is a broad resonance, which is typical for overlapping $\mathrm{e}_{\mathrm{g}}$ states in contrast to the well separated, narrow $\mathrm{t}_{2 \mathrm{~g}}$ states (FWHM V7=1.6 eV, FWHM V6=0.9 eV). Thus we normalised the sum of the intensity of V1-V6 on the remaining intensity of $\mathrm{V} 7$, thereby estimating the $\mathrm{t}_{2 \mathrm{~g}} / \mathrm{e}_{\mathrm{g}}$ ratio.

The different response of the catalyst surface on the applied conditions is very obvious when the $t_{2 g} / e_{g}$ ratio is compared (Fig. 4C). A strong increase in favour of the $t_{2 g^{-}}$ like states was observed under vacuum conditions at $400^{\circ} \mathrm{C}$ $(156 \%)$, but a decrease in the $\mathrm{t}_{2 \mathrm{~g}} / \mathrm{e}_{\mathrm{g}}$-ratio was detected when the catalyst was heated in pure oxygen ( $85 \%$ of the initial value). The same analysis of the $\mathrm{V} \mathrm{L}_{3}$-NEXAFS recorded under $n$-butane oxidation conditions revealed a small increase of the $t_{2 g}$-states relative to the $e_{g}$-states to $117 \%$ of its initial value. Cooling down the catalyst to room temperature caused a decrease of this ratio close to its initial value (97\% for $n$-butane/oxygen, $95 \%$ for pure oxygen) in the experiment with a gaseous atmosphere while the $t_{2 \mathrm{~g}} / \mathrm{e}_{\mathrm{g}}$ ratio was still increased to $138 \%$ after heating in vacuum. The evolution of the $t_{2 \mathrm{~g}} / \mathrm{e}_{\mathrm{g}}$ ratio during heating / cooling cycles (light bar / dark bar) remained inside the margin of the error bars both for vacuum conditions and for pure oxygen. Thus, a significant dependence of this ratio on the temperature within the $90 \%$ confidence interval could not be proven by the experimental data under these conditions. The situation is different for the temperature cycle in the mixture of $n$-butane and oxygen. There, a clear trend of the mean became visible when the temperature was cycled between room temperature and the reaction temperature of $400{ }^{\circ} \mathrm{C}$. This is shown in Fig. $4 \mathrm{C}+2 \mathrm{C}$. This suggests that the amount of empty $\mathrm{t}_{2 \mathrm{~g}}$-states is increased relative to the states with $e_{g}$ character under $n$-butane oxidation conditions. This corresponds to a redistribution of d-electrons at the surface states, which means a change in the character of the V-O bonding or in other words, in the present case of a covalent bond, a re-hybridisation of the metal-ligand orbitals. The interaction of adsorbate molecules with these states or a transfer of electrons due to a change in the oxidation state of vanadium will of course strongly affect the $\mathrm{V}$ d-states, too. Otherwise, in separate in situ XPS experiments no major $\mathrm{V}$ oxidation state changes were observed under aerobic $n$-butane oxidation conditions [15].

The NEXAFS of the surface showed remarkable differences when the catalyst was heated in pure oxygen compared to the state when the sample was treated at $n$-butane oxidation conditions and in vacuum. In pure oxygen there was a strong, non-reversible intensity increase of V6 and the high photon energy side of the spectrum (area of $\mathrm{e}_{\mathrm{g}}$-like states, respectively $\sigma$-bonds) gained intensity relative to the low energy side (area of $t_{2 g}$-like states, respectively $\pi$ bonds) (Fig. 4B+C). Furthermore, all resonance intensities $\mathrm{V} 1-\mathrm{V} 7$ were affected more or less by the heating in pure oxygen in contrast to the heating in $n$-butane / oxygen when other resonance intensities than V5 / V6 showed almost no response to the heating procedure. This observation leads to the conclusion that another phase is formed during the heating in oxygen, during which the overall NEXAFS is shifted to higher photon energies. We calculated a difference spec trum at the $\mathrm{V} \mathrm{L}_{3}$-edge of the normalised NEXAFS before and after the heating procedure. The resulting spectrum can be found in Fig. 5a. Although the details of the spectrum depend on the normalisation prior to the subtraction, it becomes clear that the spectral weight of the difference spectrum (a) is shifted to higher photon energies compared to the spectrum of the VPO catalyst before the heating in oxygen (e). This is typical for $\mathrm{V}^{5+}$ species $[25,27]$. The NEXAFS of the $\mathrm{V}^{5+}$-phases $\mathrm{VOPO}_{4} * 2 \mathrm{H}_{2} \mathrm{O}(\mathrm{b}), \beta-\mathrm{VOPO}_{4}$ (c) and $\mathrm{V}_{2} \mathrm{O}_{5}$ (d) are also shown in Fig. 5 for comparison. An unambiguous identification of the species is difficult but it becomes evident that a $\mathrm{V}^{5+}$ species was formed. The formation of $\mathrm{V}^{5+}$ phases in oxygen is also described in the literature $[10,28,29]$.

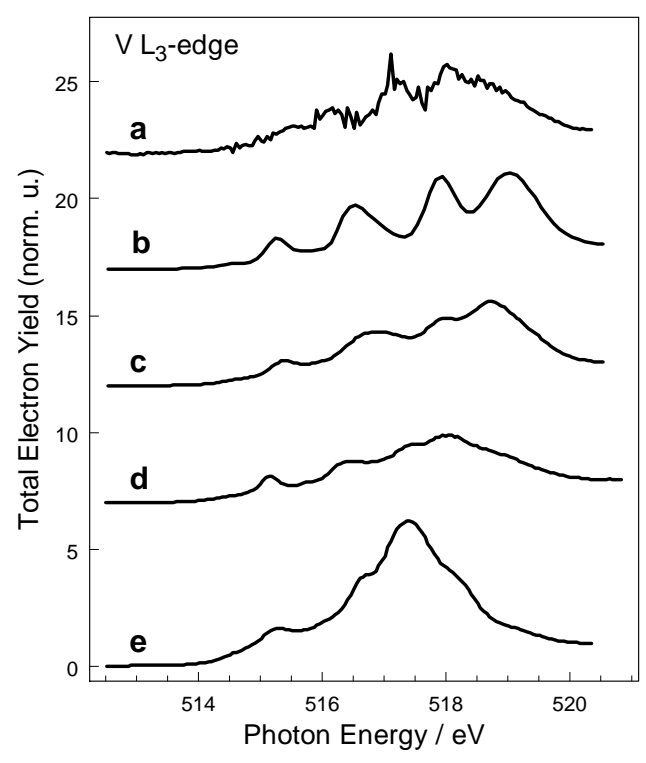

Figure 5: $\mathrm{V} \mathrm{L}_{3}$-NEXAFS of different VPO phases: a): difference spectrum after the VPO catalyst has been heated in $0.4 \mathrm{mbar}_{2}$ at $400{ }^{\circ} \mathrm{C}$ for $180 \mathrm{~min}$. For comparison the $\mathrm{V} \mathrm{L}_{3}$ absorption edge of the $\mathrm{V}^{5+}$ phases b): $\mathrm{VOPO}_{4} * 2 \mathrm{H}_{2} \mathrm{O}$, c): $\left.\beta-\mathrm{VOPO}_{4}, \mathbf{d}\right): \mathrm{V}_{2} \mathrm{O}_{5}$ and of the VPO catalyst before the heating in oxygen (e) are shown. The spectra have been background subtracted and normalised to the same edge height.

From the above it can be concluded that the VPO catalyst showed a specific response to the chemical potential of the applied atmosphere when it was heated to reaction temperature. The reversibility of the observed intensity changes is also controlled by the nature of the gas phase.

The variation of the V5 and V6 resonance energy position during the heating / cooling cycles under the different conditions is shown in Fig. 6. The resonance positions were 
slightly shifted to smaller photon energies under all applied conditions when the catalyst was heated to $400{ }^{\circ} \mathrm{C}$. The resonance position shift of $\mathrm{V} 6$ was stronger than the energy
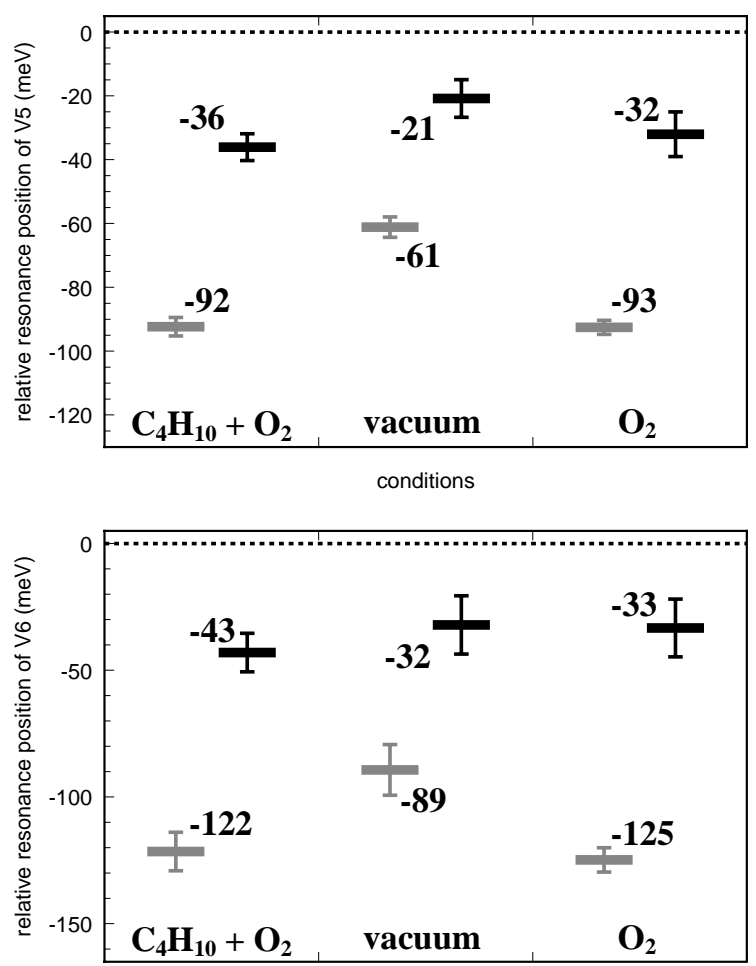

conditions

Figure 6: Averaged value of the relative photon energy position of V5 (A) and V6 (B). The catalyst was heated to $400{ }^{\circ} \mathrm{C}$ (light bar) and cooled down to room temperature (dark bar) under different conditions: $\mathrm{C}_{4} \mathrm{H}_{10}+\mathrm{O}_{2}$, vacuum, and $\mathrm{O}_{2}$. The initial energy position of the resonances prior to the heating procedure was taken as 0 (dashed line). The error bar indicates the $90 \%$ confidence interval of the data point.

shift of V5 in agreement with previous observations for VPO catalysts prepared by the aqueous preparation route [15]. This shift was a bit less pronounced under vacuum conditions. The resonance positions shifted back to a value close to the initial one when the sample was cooled down to room temperature, although not completely.

There are various explanations for a resonance position shift in XAS. In transition metal oxides the resonance position shifts can occur due to a change in the electrostatic potential of the absorbing metal atom. This fact is used to conclude from the resonance energy position upon the valence of the metal ion. A linear shift to lower energies with decreasing oxidation state is observed $[30,25]$ as described before in connection with the discussion of Fig. 5. Otherwise, recent in situ XPS investigation of VPO catalyst did not indicate a major change of the vanadium valence under aerobic $n$-butane oxidation conditions [15]. Vinogradov et al. found a shift of the centre of mass of the $\mathrm{Fe} \mathrm{L}_{3}$ NEXAFS for iron compounds with the same formal oxidation state and geometry but a different ligand [31]. They studied ligands with different ionicity. They concluded that the reason for the shift is the difference in hybridisation between the metal $3 \mathrm{~d}$ molecular orbital and the $2 \mathrm{p}$ ligand orbital. Thus the observed shift in the V5 and V6 resonance position in our experiment supports rather the interpretation of a variation of the $\mathrm{V}-\mathrm{O}$ bond character under reaction conditions than a $\mathrm{V}$ valence change. The evolution of the $t_{2 g} / e_{g}$ ratio given in Fig. $2 C$ suggests such a change in the bond character, too.

The local nature of the NEXAFS allows a detailed spectroscopic insight into specific V-O bonds of the material. It is feasible to deconvolute at least qualitatively the overall spectral function at the $\mathrm{V} \mathrm{L}_{3}$-absorption edge into main contributions from differently coordinated oxygen $[15,25]$. The basic structural units of vanadyl pyrophosphate are twinned highly distorted octahedra as depicted in Fig. 7 [32]. These octahedra are isolated and connected from other $\mathrm{VO}_{6}$ octahedra pairs by $\mathrm{P}_{2} \mathrm{O}_{7}$ double tetrahedra. In [25] we suggested a linear relationship between the energy position of several absorption resonances and the bond length of the participating atoms. From this empirical bond length / resonance position relationship it can be concluded that resonance V6 represents mainly the electron density of the vanadyl group in VPO $\left(\mathrm{V}-\mathrm{O}_{\text {vanadyl }}\right)$.

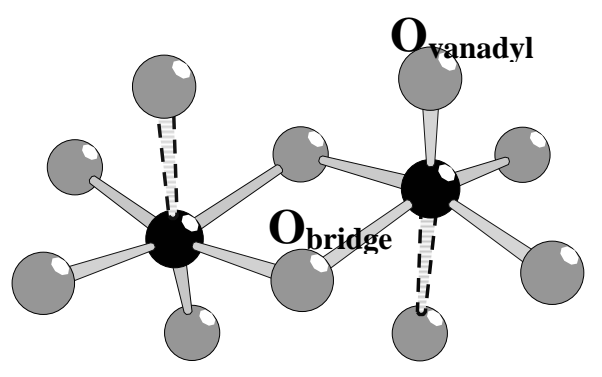

Figure 7: Model of structural units of vanadyl pyrophosphate according to Nguyen et al. [32]. Twinned highly distorted $\mathrm{VO}_{6}$ octahedra are shown with central vanadium atoms (black) surrounded by oxygen (grey).

The intensity of resonance V6 might be influenced by $\mathrm{e}_{\mathrm{g}}$ states, too. A small but finite $\mathrm{t}_{2 \mathrm{~g}}-\mathrm{e}_{\mathrm{g}}$ configuration mixing is a measure of the octahedral distortion, which reduces the local symmetry at the $\mathrm{V}$ sites. A stronger distortion of the $\mathrm{VO}_{6}$ octahedron during reaction conditions would cause a loss of the separation of the $e_{g}$ and $t_{2 g}$ states. The $e_{g}$ states would extend over a larger energy range and overlap stronger with the $t_{2 g}$ like states. This will result in an apparent increase of V6 and a decrease of V7 and therefore an increase of the $t_{2 \mathrm{~g}} / \mathrm{e}_{\mathrm{g}}$ ratio which is calculated by (V1V6)/V7 without a change in the occupation number of these states. The known expulsion of phosphorous at the surface and an oxide linkage different from the double $\mathrm{VO}_{6}$ octa- 
hedra of $(\mathrm{VO})_{2} \mathrm{P}_{2} \mathrm{O}_{7}$ might be the origin for this distortion under working conditions.

The origin of resonance $\mathrm{V} 5$ is less obvious. No identification of the $\mathrm{V}-\mathrm{O}$ bond distance in $(\mathrm{VO})_{2} \mathrm{P}_{2} \mathrm{O}_{7}$ can be given. There can be found an alike bond in $\mathrm{V}_{2} \mathrm{O}_{5}$ between the central vanadium atom and the bridging, planar oxygen amounting to in -plane distances of $1.779 \AA$ within the octahedra chains ( $\left.\mathrm{V}-\mathrm{O}_{\text {bridge}}\right)$.

The active surface of a VPO catalyst is likely nonstoichimotric both in the elemental composition $(\mathrm{P} / \mathrm{V}$ ratio $>1$ ) and the redox chemistry. Although the role of $\mathrm{V}^{5+}$ sites is discussed controversially in literature the presence of dispersed $\mathrm{V}^{5+}$ sites seems to be beneficial for the performance of the catalyst. $\mathrm{V}^{5+}$ microdomains at the surface have been made responsible for this effect [33]. A kinetic relationship between $\mathrm{V}^{5+}$ centres and the yield of MA under unsteady n-butane oxidation conditions was demonstrated by Coulston et al. [34]. It was shown in our paper by the oxidation of VPO in pure oxygen, that NEXAFS is a sensitive tool to detect changes in the $\mathrm{V}$ valence. There was however no unambiguous evidence that the $\mathrm{V}$ valence changed under steady state aerobic $n$-butane oxidation conditions. The overall $\mathrm{V} \mathrm{L}_{3}$-peak position agreed with an oxidation state slightly higher than +4 . Minor changes in the $\mathrm{V}$ valence of the outermost surface under reaction conditions might be undetectable with NEXAFS. A profound analysis of the NEXAFS data concerning the presence and identification of minor $\mathrm{V}^{5+}$ species remains inconclusive without having access to pure phases. These reference phases might be unavailable if the distortion of the vanadyl pyrophosphate occurs only as transient phenomenon under reaction conditions.

Otherwise, it became clear from our data, that there is a substantial flexibility in the local electronic structure of the VPO surface under reaction conditions. A biphasic near-surface region of the active catalyst offering V-O bonds with different hybridisation could provide this flexibility. The interplay of these species in the catalytic process might be formally assigned to $\mathrm{V}^{4+} / \mathrm{V}^{5+}$ redox couple.

\section{Conclusions}

Our extensive investigation of a VPO catalyst under different ambient conditions indicates that the catalyst surface clearly responded to the applied conditions. It became

\section{References}

[1] R.L. Bergmann, N.W. Frisch, US Patent 3,393,368 (1968) assigned to Princeton Chemical Research.

[2] T.R. Felthouse, J.C. Burnett, S.F. Mitchell, M.J. Mummy, Kirk-Othmer Encyclopedia of Chemical Technology, Wiley, New York, 1995

[3] G. Centi (Ed.) Vanadyl Pyrophosphate Catalysts. In Catalysis Today, Elsevier: Amsterdam, 1993; Vol. 16, Part 1.

[4] G. Centi and S. Perathoner, Current Opinion in Solid State \& Materials Science 4 (1999) 74.

[5] S. Albonetti, F. Cavani, F. Trifiro, Catal. Rev.-Sci. \& Eng. 38 (1996) 413. evident that results about the electronic structure of the catalyst surface obtained under conditions other than the reaction atmosphere should be considered with reservation. This problem cannot be solved by a pre-treatment of the material, because most of the changes of the electronic structure under $n$-butane oxidation conditions described in this report are reversible and disappeared instantaneously when the catalyst was cooled back to room temperature. This is in contrast to the result when the catalyst was heated in pure oxygen with the formation of a stable $\mathrm{V}^{5+}$ species. In that case, the same result could be obtained after an oxidative pre-treatment of the catalyst $[10,28]$. It was shown that neither the temperature alone nor beam damage can explain the observed variation of the NEXAFS of the catalyst under $n$-butane oxidation conditions. The formation of the specific metastable state of the electronic surface structure required specific boundary conditions (temperature, gas phase composition). It is likely that the hybridisation of the $\mathrm{V}-\mathrm{O}$ bonds provides the desired electronic flexibility. It can be concluded that the character of specific V-O bonds $\left(\mathrm{V}-\mathrm{O}_{\text {vanadyl }}\right.$, in-plane $\left.\mathrm{V}-\mathrm{O}_{\text {bridge }}\right)$ of the surface are altered in the active state of the catalyst. The relevance of the subtle changes of the electronic surface structure for the catalytic activity has been demonstrated recently by simultaneously recording the VPO NEXAFS and the formation of MA under steady $n$-butane oxidation conditions $[15,35]$.

No major $\mathrm{V}$ oxidation state change could be detected under steady state aerobic $n$-butane oxidation conditions. X-ray photoelectron spectroscopy (XPS) can provide additional, quantitative information about the $\mathrm{V}$ valence. Therefore, we will apply in situ XPS to reveal more detailed information about the role of $\mathrm{V}^{5+}$ centres at the surface of VPO catalysts in the $n$-butane oxidation reaction in our future work.

\section{Acknowledgement}

This work was carried out in co-operation with the groups of G. Hutchings and J.C. Volta. The authors thank the BESSY staff for their continual support during the XAS measurements at the synchrotron in Berlin. The work was supported by SFB 546 of the Deutsche Forschungsgemeinschaft (DFG) and by EPSRC (ATHENA).

[6] J.C. Volta, Chemistry 3 (2000) 717.

[7] B. Grzybowska-Swierkosz, Top. Catal. 11 (2000) 23.

[8] J. C. Volta, Catal. Today 32 (1996) 29.

[9] E. Bordes, Catal. Today 1 (1987) 499.

[10] L.M. Cornaglia and E.A. Lombardo, Appl. Catal. A-General 127 (1995) 138.

[11] S. Albonetti, F. Cavani, F. Trifirò, P. Venturoli, G. Calestani, M. López Granados, J.L.G. Fierro, J. Catal. 160 (1996) 52.

[12] V.V. Guliants, J.B. Benzinger, N.Y. Sundaresan, I.E. Wachs, Catal. Lett. 32 (1995) 379.

[13] G.J. Hutchings, J.A. Lopez-Sanchez, J.K. Bartley, J.M. Webster, A. Burrows, C.J. Kiely, A.F. Carley, C. Rhodes, M. 
Hävecker, A. Knop-Gericke, R.W. Meyer, R. Schlögl, J.C. Volta, M. Poliakoff, J. Catal., 208 (2002) 197.

[14] I.E. Wachs, J.-M. Jehng, G. Deo, B.M. Weckhuysen, V.V. Guliants, J.B. Benzinger, Catal. Today 32 (1996) 47.

[15] M. Hävecker, R.W. Mayer, A. Knop-Gericke, H. Bluhm, E. Kleimenov, A. Liskowski, D. Su, R. Follath, F.G. Requejo, D.F. Ogletree, M. Salmeron, J.A. Lopez-Sanchez, J.K. Bartley, G.J. Hutchings, and R. Schlögl, J. Phys. Chem. B, 107 (2003) 4587.

[16] Knop-Gericke, M. Hävecker, T. Neisius, Th. SchedelNiedrig, Nucl. Intr. Meth. A 406 (1998) 311.

[17] M. Hävecker, A. Knop-Gericke, Th. Schedel-Niedrig, R. Schlögl, Angew. Chem. Int. Ed. 37 (1998) 206.

[18] Knop-Gericke, M. Hävecker, Th. Schedel-Niedrig, R. Schlögl, Top. Catal. 15 (2001) 27.

[19] R. Follath, F. Senf, Nucl. Instr. Meth. A 390 (1997) 388.

[20] F. Senf, K.J.S. Sawhney, Nucl. Instr. Meth. A $467 / 468$ (2001) 466.

[21] Y. Ma, C.T. Chen, G. Meigs, K. Randall, F. Sette, Phys. Rev. A 44 (1991) 1848.

[22] F. Sette, B. Sinkovic, Y.J. Ma, C.T. Chen, Phys. Rev. B 39 (1989) 11125.

[23] F.M.F. de Groot, J.C. Fuggle, B.T. Thole, G.A. Sawatzky, Phys. Rev. B 41 (1990) 928.

[24] C.J. Ballhausen, Introduction to Ligand Field Theory, McGrawHill, New York, 1962.
[25] M. Hävecker, A. Knop-Gericke, R. W. Mayer, M. Fait, H. Bluhm, R. Schlögl, J. Electron Spectrosc. Relat. Phenom. 125 (2002) 79.

[26] V. Eyert, K.-H. Höck, Phys. Rev. B, 1998, 57, 12727.

[27] M. Abbate, H. Pen, M.T. Czyzyk, F.M.F. de Groot, J.C. Fuggle, Y. Ma, C.T. Chen, F. Sette, A. Fujimori, Y. Ueda, K.J. Kosuge, J. Electron Spetrosc. Relat. Phenom. 62 (1993) 185.

[28] Xiao-Feng Huang, Biao-Hua Chen, Bai-Jian Liu, P.L. Silveston, Cheng-Yue Li, Catal. Today 74 (2002) 121.

[29] V.V. Guliants, J.B. Benzinger, S. Sundaresan, I.E. Wachs, J.M. Jehng, J.E. Roberts, Catal. Today 28 (1996) 275.

[30] J.G. Chen, Surf. Sci. Rep. 30 (1997) 1.

[31] A.S. Vinogradov, A.B. Preobrajenski, S.A. Krasnikov, T. Chassé, R. Szargan, A. Knop-Gericke, R. Schlögl, P. Bressler, Surf. Rev. Lett. 9 (2002) 359.

[32] P.T. Nguyen, R.D. Hoffman, A.W. Sleight, Mater. Res. Bull. 30 (1995) 1055.

[33] M. Abon, J.M. Herrman, J.C. Volta, Catal. Today 71 (2001) 121.

[34] G.W. Coulston, S.R. Bare, H. Kung, K. Birkeland, G.K. Bethke, R. Harlow, N. Herron, P.L. Lee, Science 267 (1997) 191.

[35] H. Bluhm, M. Hävecker, E. Kleimenov, A. Knop-Gericke, A. Liskowski, R. Schlögl, D.S. Su, Top. Catal. 23 (2003) 99. 